\title{
On the wellposedness of the Chaboche model
}

\author{
Martin Brokate* \\ Mathematisches Seminar \\ Universität Kiel \\ 24098 Kiel \\ Germany
}

\author{
Pavel Krejčí $i^{\dagger \ddagger}$ \\ Institute of Mathematics \\ Academy of Sciences \\ Žitná 25 \\ 11567 Praha \\ Czech Republic
}

\begin{abstract}
We formally state and prove the wellposedness and the local Lipschitz continuity of the multisurface stress-strain law of nonlinear kinematic hardening type due to Chaboche within the space of time-dependent tensor-valued absolutely continuous functions. The results also include the more general case of a continuous family of auxiliary surfaces.
\end{abstract}

\section{Introduction}

In rate independent plasticity, the Prandtl-Reuß model constitutes the basic model for the stress-strain law. Here, the elastic region $Z$ is bounded by a yield surface $\partial Z$. Throughout this paper, we will assume the yield surface to be a sphere of radius $r$ in the space of deviatoric stresses. If loading occurs while the stress deviator $\sigma_{d}$ lies on the yield surface, there is plastic flow with a plastic strain rate $\dot{\varepsilon}^{p}$ proportional to the outer normal to $\partial Z$ in $\sigma_{d}$. It has been known from experiments for a long time that for many materials the yield surface undergoes changes which depend upon the history of the loading process. In the Melan-Prager model which dates back to [12], [13], nowadays called linear kinematic hardening, the yield surface moves during plastic loading in the direction of the plastic strain rate. More sophisticated models have been developed to account for real material behaviour, in particular for the phenomenon called ratchetting. Among those, the Chaboche model [10], also called nonlinear kinematic hardening, enjoys a widespread popularity. In its standard form, it employs a finite family of auxiliary spherical surfaces. In the special case of a single auxiliary surface, assumed to be centered at 0 with radius $R$, the model is known as the Armstrong-Frederick model [1]; here, the center $\sigma^{b}$ of the yield surface, also termed the backstress, moves according to the differential equation

$$
\dot{\sigma}^{b}=\gamma\left(R \dot{\varepsilon}^{p}-\sigma^{b}\left|\dot{\varepsilon}^{p}\right|\right)
$$

*Supported by the BMBF, Grant No. 03-BR7KIE-9, within "Anwendungsorientierte Verbundprojekte auf dem Gebiet der Mathematik".

†Supported by the BMBF during his stay at Kiel.

${ }^{\ddagger}$ Partially supported by the Grant Agency of the Czech Republic under Grant No. 201/95/0568. 
for some constant $\gamma>0$, see Figure 1. (In the Melan-Prager model, the term $-\sigma^{b}\left|\dot{\varepsilon}^{p}\right|$ is omitted.)



Figure 1: The Armstrong-Frederick model.

In the Chaboche model, the backstress $\sigma^{b}$ is decomposed into a sum

$$
\sigma^{b}=\sum_{k \in I} \sigma_{k}^{b}
$$

where each constituent $\sigma_{k}^{b}$ satisfies an equation of type (1.1), namely

$$
\dot{\sigma}_{k}^{b}=\gamma(k)\left(R(k) \dot{\varepsilon}^{p}-\sigma_{k}^{b}\left|\dot{\varepsilon}^{p}\right|\right), \quad k \in I .
$$

In the standard Chaboche model, the index set $I$ is finite; we will allow an arbitrary measure space and thus include the case of a continuous family of auxiliary surfaces.

Figure 2 shows the rheological structure of the model. It visualizes the relations between the various variables which occur in the model, stated formally in (2.5) - (2.12) below. The element $\mathcal{E}$ refers to the linear elastic part, $\mathcal{R}$ is called the rigid plastic element and represents the variational inequality, and $\mathcal{K}_{k}$ is the element defined by (1.3). The element $\mathcal{L}$ plays a special role; it stands for the linear element $\sigma^{l}=C^{l} \varepsilon^{p}$ of the Melan-Prager model. It may or may not be included within the Chaboche model, but its presence or absence influences the asymptotic behaviour (see e.g. [7]). If we remove all nonlinear elements $\mathcal{K}_{k}$ in Figure 2, we obtain the Melan-Prager model. If we moreover delete the element $\mathcal{L}$, we arrive at the Prandtl-Reuß model.

In this paper, we prove that the Chaboche model is well posed in the space $W^{1,1}$ both in the stress controlled and in the strain controlled case by proving that the defining equations and inequalities of the Chaboche model (see (2.5) - (2.12) below) lead to operators

$$
\varepsilon=\mathcal{F}(\sigma), \quad \sigma=\mathcal{G}(\varepsilon),
$$


which are well defined and Lipschitz continuous on their appropriate domains of definition. In doing this, we consider the stress-strain law in isolation, that is, we do not study the boundary value problems which arise from the coupling with the balance equations. For the proof we utilize the method of [2]. There we have introduced an auxiliary variable $u$ in order to reformulate the model equations such that the unknown functions of Figure 2 appear only in terms of $\left|\dot{\varepsilon}^{p}\right|$ and $\sigma_{d}^{p}$. The analysis is based on the concept of hysteresis operators, that is, of operators which are rate-independent as well as causal, see e.g. [14], $[8],[9],[3]$.

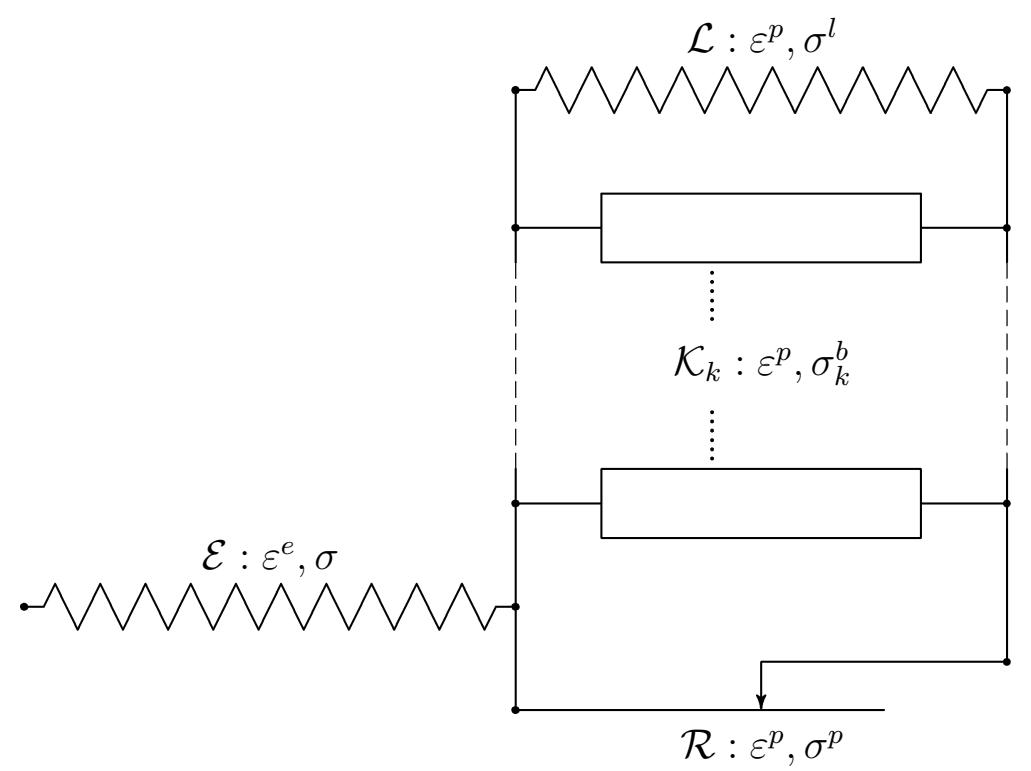

Figure 2: The rheological stucture of the Chaboche model. 


\section{Model Formulation and Main Result}

We first fix some basic tensor notation. By $\mathbb{T}$, we denote the space of symmetric $N \times N$ tensors endowed with the usual scalar product and the associated norm

$$
\langle\tau, \eta\rangle=\sum_{i, j=1}^{N} \tau_{i j} \eta_{i j}, \quad|\tau|=\sqrt{\langle\tau, \tau\rangle},
$$

For $\tau \in \mathbb{T}$, we define its trace $\operatorname{Tr} \tau$ and its deviator $\tau_{d}$ by

$$
\operatorname{Tr} \tau=\sum_{i=1}^{N} \tau_{i i}=\langle\tau, \delta\rangle, \quad \tau_{d}=\tau-\frac{\operatorname{Tr} \tau}{N} \delta,
$$

where $\delta=\left(\delta_{i j}\right)$ stands for the Kronecker symbol. We denote by

$$
\mathbb{T}_{d}=\{\tau: \tau \in \mathbb{T}, \operatorname{Tr} \tau=0\}, \quad \mathbb{T}_{d}^{\perp}=\{\tau: \tau=\lambda \delta, \lambda \in \mathbb{R}\},
$$

the space of all deviators respectively its orthogonal complement. We understand stress and strain as time-dependent tensor-valued functions which are absolutely continuous,

$$
\sigma, \varepsilon \in W^{1,1}\left(t_{0}, t_{1} ; \mathbb{T}\right):=\left\{\tau\left|\tau:\left[t_{0}, t_{1}\right] \rightarrow \mathbb{T},\|\tau\|_{1,1}=\right| \tau\left(t_{0}\right)\left|+\int_{t_{0}}^{t_{1}}\right| \dot{\tau}(t) \mid d t<\infty\right\}
$$

As we study the stress-strain law in isolation, we do not consider the space dependence. In this terminology, the Chaboche model takes on the form

$$
\begin{gathered}
\sigma=\sigma^{b}+\sigma^{p}, \quad \varepsilon=\varepsilon^{e}+\varepsilon^{p}, \quad \varepsilon^{p}(t) \in \mathbb{T}_{d} \quad \forall t, \\
\left\langle\dot{\varepsilon}^{p}, \sigma_{d}^{p}-\tilde{\sigma}\right\rangle \geq 0, \quad \forall \tilde{\sigma} \in \mathbb{T}_{d},|\tilde{\sigma}| \leq r, \\
\left|\sigma_{d}^{p}\right| \leq r, \\
\sigma=A \varepsilon^{e}, \\
\sigma^{b}(t)=\int_{I} \sigma_{k}^{b}(t) d \nu(k)+\nu^{l} \sigma^{l}(t) \quad \forall t, \\
\dot{\sigma}_{k}^{b}=\gamma(k)\left(R(k) \dot{\varepsilon}^{p}-\sigma_{k}^{b}\left|\dot{\varepsilon}^{p}\right|\right), \quad \text { for all } k \in I, \\
\sigma^{l}=C^{l} \varepsilon^{p},
\end{gathered}
$$

Throughout this paper, we assume the data to have the following properties.

\section{Assumption 2.1}

(i) $I$ is a measure space, $\nu$ is a finite nonnegative measure on $I$, the numbers $\nu^{l}, C^{l}$ and functions $R \in L_{\nu}^{1}(I), \gamma \in L_{\nu}^{\infty}(I)$ satisfy $\nu^{l}, R, \gamma \geq 0, C^{l}>0, \int_{I} R(k) d \nu(k)>0$ and

$$
0<\gamma_{\min } \leq \gamma(k) \leq \gamma_{\max }, \quad \text { for all } k \in I
$$

(ii) The initial values in (2.12) satisfy

$$
\begin{gathered}
\sigma_{0}^{p} \in \mathbb{T}_{r}^{p}=\left\{\tau: \tau \in \mathbb{T},\left|\tau_{d}\right| \leq r\right\} \\
\sigma_{0}^{b} \in \mathcal{T}^{b}=\left\{f\left|f \in L_{\nu}^{1}\left(I ; \mathbb{T}_{d}\right),\right| f(k) \mid \leq R(k) \text { a.e. }\right\}
\end{gathered}
$$


(iii) $A: \mathbb{T} \rightarrow \mathbb{T}$ is linear, symmetric and positive definite.

We also introduce the constants

$$
\Gamma_{i}=\int_{I} \gamma(k)^{i} R(k) d \nu(k), \quad i=0,1,2,3 .
$$

\section{Remark 2.2}

(i) If the index set $I$ is finite, say $I=\{1, \ldots, K\}$, and if $\nu$ is chosen to be the counting measure, that is, $\nu(J)$ equals the number of elements in $J$ for every subset $J$ of $I$, then we obtain the standard formulation of the multisurface Chaboche model with $K$ auxiliary (limiting) surfaces, namely

$$
\sigma^{b}=\sum_{k=1}^{K} \sigma_{k}^{b}
$$

In this case, the model (2.5) - (2.12) is identical with the one discussed in ([10], Section 5.4.4), nonlinear kinematic case, if we change the notation according to

$$
k \hat{=} l, \quad \sigma_{k}^{b} \hat{=} \mathbf{X}_{l}, \quad \gamma(k) \hat{=} \sqrt{\frac{2}{3}} \gamma_{l}, \quad \gamma(k) R(k) \hat{=} \frac{2}{3} C_{l} .
$$

(ii) If we have $K=1$ in (i), or if we choose $\gamma(k) \equiv \gamma$ and $R(k) \equiv R / \nu(I)$ to be constant, the Chaboche model reduces to the model of Armstrong and Frederick [1]

$$
\dot{\sigma}^{b}=\gamma\left(R \dot{\varepsilon}^{p}-\sigma^{b}\left|\dot{\varepsilon}^{p}\right|\right) .
$$

(iii) If $d \nu(k)=g(k) d \lambda(k)$ for some function $g$, that is, if the measure $\nu$ has a density with respect to the Lebesgue measure $\lambda$, we obtain a version of the Chaboche model with a continuous one parameter family of backstresses respectively auxiliary surfaces.

We formulate our main results. For the strain controlled case, we assume Hooke's law for the linear elastic part, that is,

$$
A \varepsilon=2 \mu \varepsilon+\lambda \operatorname{Tr}(\varepsilon) \delta
$$

where $\lambda, \mu>0$ denote the Lamé constants.

\section{Theorem 2.3 (Wellposedness, Strain Controlled Case)}

Let Assumption 2.1 as well as (2.20) hold. Then the system (2.5) - (2.12) defines an operator

$$
\begin{gathered}
\sigma=\mathcal{G}\left(\varepsilon ; \sigma_{0}^{p}, \sigma_{0}^{b}\right), \\
\mathcal{G}: W^{1,1}\left(t_{0}, t_{1} ; \mathbb{T}\right) \times \mathbb{T}_{r}^{p} \times \mathcal{T}^{b} \rightarrow W^{1,1}\left(t_{0}, t_{1} ; \mathbb{T}\right),
\end{gathered}
$$

which satisfies the Lipschitz condition

$$
\left\|\mathcal{G}\left(\varepsilon ; \sigma_{0}^{p}, \sigma_{0}^{b}\right)-\mathcal{G}\left(\bar{\varepsilon} ; \bar{\sigma}_{0}^{p}, \bar{\sigma}_{0}^{b}\right)\right\|_{1,1} \leq L(K)\left(\|\varepsilon-\bar{\varepsilon}\|_{1,1}+\left|\sigma_{0}^{p}-\bar{\sigma}_{0}^{p}\right|+\left\|\sigma_{0}^{b}-\bar{\sigma}_{0}^{b}\right\|_{L_{\nu}^{1}\left(I ; \mathbb{T}_{d}\right)}\right),
$$

where the Lipschitz constant is uniform over subsets $\left\{\left(\varepsilon, \sigma_{0}^{p}, \sigma_{0}^{b}\right):\|\varepsilon\|_{1,1} \leq K\right\}$ of the domain of definition of $\mathcal{G}$. 
We now consider the stress controlled case. If $\nu^{l}=0$, that is, if the Melan-Prager element is absent, our choice (2.12) of initial conditions restricts the initial value $\sigma\left(t_{0}\right)$ of the stress; on the other hand, there has to be an initial condition

$$
\varepsilon^{p}\left(t_{0}\right)=\varepsilon_{0}^{p}
$$

for the plastic strain. This setting also works for the case $\nu^{l}>0$, the restriction being

$$
\sigma\left(t_{0}\right)=\sigma_{0}^{p}+\int_{I} \sigma_{0}^{b}(k) d \nu(k)+\nu^{l} C^{l} \varepsilon_{0}^{p} .
$$

In the case $\nu^{l}=0$, the description of the domains where the Lipschitz constant is uniform involves the number

$$
\beta=\frac{1}{\gamma_{\min }}+\frac{r \gamma_{\max }}{\Gamma_{2}}
$$

\section{Theorem 2.4 (Wellposedness, Stress Controlled Case)}

Let Assumption 2.1 hold.

(Case $\nu^{l}>0$.) The system (2.5) - (2.12), (2.24) defines an operator

$$
\varepsilon=\mathcal{F}\left(\sigma ; \sigma_{0}^{p}, \sigma_{0}^{b}, \varepsilon_{0}^{p}\right), \quad \mathcal{F}: D_{\sigma} \rightarrow W^{1,1}\left(t_{0}, t_{1} ; \mathbb{T}\right),
$$

where $D_{\sigma} \subset W^{1,1}\left(t_{0}, t_{1} ; \mathbb{T}\right) \times \mathbb{T}_{r}^{p} \times \mathcal{T}^{b} \times \mathbb{T}_{d}$ is the subset of quadruples which satisfy (2.25). Moreover, $\mathcal{F}$ satisfies on $D_{\sigma}$ the Lipschitz condition

$$
\begin{aligned}
& \left\|\mathcal{F}\left(\sigma ; \sigma_{0}^{p}, \sigma_{0}^{b}, \varepsilon_{0}^{p}\right)-\mathcal{F}\left(\bar{\sigma} ; \bar{\sigma}_{0}^{p}, \bar{\sigma}_{0}^{b}, \bar{\varepsilon}_{0}^{p}\right)\right\|_{1,1} \\
& \quad \leq L(K)\left(\|\sigma-\bar{\sigma}\|_{1,1}+\left|\sigma_{0}^{p}-\bar{\sigma}_{0}^{p}\right|+\left\|\sigma_{0}^{b}-\bar{\sigma}_{0}^{b}\right\|_{L_{\nu}^{1}\left(I ; \mathbb{T}_{d}\right)}+\left|\varepsilon_{0}^{p}-\bar{\varepsilon}_{0}^{p}\right|\right),
\end{aligned}
$$

where the Lipschitz constant is uniform over subsets $\left\{\left(\sigma, \sigma_{0}^{p}, \sigma_{0}^{b}, \varepsilon_{0}^{p}\right):\|\sigma\|_{1,1} \leq K\right\}$ of the domain of definition of $\mathcal{F}$.

(Case $\left.\nu^{l}=0.\right)$ For every $\kappa>0$, let $D_{\sigma, \kappa}$ be the subset of $D_{\sigma}$ where the two conditions

$$
\begin{gathered}
\left|\int_{I} \gamma(k) \sigma_{0}^{b}(k) d \nu(k)\right| \leq \Gamma_{1}(1-\kappa), \\
\left\|\sigma_{d}\right\|_{\infty} \leq \Gamma_{0}+r-\Gamma_{1} \beta \kappa,
\end{gathered}
$$

hold, the number $\beta$ being defined in (2.26). Then $\mathcal{F}$ has the properties as stated above on the domains $D_{\sigma, \kappa}$ instead of $D_{\sigma}$; in particular, the Lipschitz constant also depends on $\kappa$.

A well known example (see [10], or Example 3.5 in [2]) shows that the bound $\left\|\sigma_{d}\right\|_{\infty}<$ $\Gamma_{0}+r$ in (2.30) cannot be improved.

The basic idea of the proof of the two theorems above is the same as in [2]. We replace the two unknown functions $\varepsilon^{p}$ and $\sigma_{d}^{p}$ by a single auxiliary function $u$, namely

$$
u=C \varepsilon^{p}+\sigma_{d}^{p},
$$

where $C>0$ is a suitably chosen constant. In fact, both functions $\varepsilon^{p}$ and $\sigma_{d}^{p}$ can be expressed as

$$
\varepsilon^{p}=\frac{1}{C} \mathcal{P}\left(u ; \sigma_{0 d}^{p}\right), \quad \sigma_{d}^{p}=\mathcal{S}\left(u ; \sigma_{0 d}^{p}\right)
$$


Here, the stop operator $\mathcal{S}$ represents the solution of the evolution variational inequality

$$
\left|\sigma_{d}^{p}\right| \leq r, \quad\left\langle\dot{u}-\dot{\sigma}_{d}^{p}, \sigma_{d}^{p}-\tilde{\sigma}\right\rangle \geq 0 \quad \text { a.e. } \forall|\tilde{\sigma}| \leq r
$$

with the initial condition

$$
\sigma_{d}^{p}\left(t_{0}\right)=\sigma_{0 d}^{p}
$$

and the play operator $\mathcal{P}$ is defined by

$$
\mathcal{P}\left(u ; \sigma_{0 d}^{p}\right)=u-\mathcal{S}\left(u ; \sigma_{0 d}^{p}\right) .
$$

We refer to [2] and [9] for more details. We now derive a differential equation for $u$ where the internal variables $\sigma_{k}^{b}, \sigma^{l}, \varepsilon_{d}^{e}, \varepsilon^{p}$ appear only in terms of $\sigma_{d}^{p}$ and $\left|\dot{\varepsilon}^{p}\right|$. In the stress controlled case, we set

$$
C=\Gamma_{1}+\nu^{l} C^{l}
$$

Using the model equations, we obtain

$$
\begin{aligned}
\dot{u} & =\left(\Gamma_{1}+\nu^{l} C^{l}\right) \dot{\varepsilon}^{p}+\dot{\sigma}_{d}^{p}=\left(\Gamma_{1}+\nu^{l} C^{l}\right) \dot{\varepsilon}^{p}+\dot{\sigma}_{d}-\dot{\sigma}^{b} \\
& =\dot{\sigma}_{d}+\int_{I} \gamma(k) \sigma_{k}^{b} d \nu(k)\left|\dot{\varepsilon}^{p}\right| .
\end{aligned}
$$

In the strain controlled case, where we have assumed Hooke's law (2.20) for the linear elastic part, the backstress $\sigma^{b}$ satisfies

$$
\sigma_{d}=2 \mu \varepsilon_{d}^{e}, \quad \sigma^{b}=2 \mu \varepsilon_{d}-\left(2 \mu \varepsilon^{p}+\sigma_{d}^{p}\right)
$$

Here, we set the constant $C$ in $(2.31)$ to

$$
C=2 \mu+\Gamma_{1}+\nu^{l} C^{l}
$$

and obtain

$$
\begin{aligned}
\dot{u} & =\left(2 \mu+\Gamma_{1}+\nu^{l} C^{l}\right) \dot{\varepsilon}^{p}+\dot{\sigma}_{d}^{p}=2 \mu \dot{\varepsilon}^{p}+2 \mu \dot{\varepsilon}_{d}^{e}-\dot{\sigma}^{b}+\left(\Gamma_{1}+\nu^{l} C^{l}\right) \dot{\varepsilon}^{p} \\
& =2 \mu \dot{\varepsilon}_{d}+\int_{I} \gamma(k) \sigma_{k}^{b} d \nu(k)\left|\dot{\varepsilon}^{p}\right|
\end{aligned}
$$

As it is well known, one can easily eliminate the unknowns $\sigma_{k}^{b}$ with the variations of constants formula. Using the basic identity

$$
\dot{\varepsilon}^{p}=\frac{\sigma_{d}^{p}}{r}\left|\dot{\varepsilon}^{p}\right|,
$$

the differential equation (2.10) for the backstresses becomes

$$
\dot{\sigma}_{k}^{b}=\gamma(k)\left(\frac{R(k)}{r} \sigma_{d}^{p}-\sigma_{k}^{b}\right)\left|\dot{\varepsilon}^{p}\right|, \quad k \in I .
$$

For later use, we will write down the solution formula in terms of the play and stop operator with the abbreviated notation

$$
\xi=\mathcal{P}\left(u ; \sigma_{0 d}^{p}\right), \quad x=\mathcal{S}\left(u ; \sigma_{0 d}^{p}\right), \quad \xi, x:\left[t_{0}, t_{1}\right] \rightarrow \mathbb{T}_{d} .
$$


The function

$$
V(t)=\operatorname{Var}_{\left[t_{0}, t\right]} \xi \quad\left(=\int_{t_{0}}^{t}|\dot{\xi}(\tau)| d \tau, \quad \text { if } \xi \in W^{1,1}\left(t_{0}, t_{1} ; \mathbb{T}_{d}\right)\right)
$$

represents the accumulated plastic strain, scaled by a constant factor. If we set

$$
W_{k}(t)=\exp \left(\frac{\gamma(k)}{C} V(t)\right)
$$

the backstresses can be expressed as

$$
\sigma_{k}^{b}(t)=\exp \left(-\frac{\gamma(k)}{C} V(t)\right)\left(\sigma_{0}^{b}(k)+\int_{t_{0}}^{t} \frac{R(k)}{r} x(\tau) d W_{k}(\tau)\right) .
$$

Thus, for the stress as well as for the strain controlled case, the auxiliary function $u$ satisfies the equation

$$
\dot{u}=\dot{\theta}+\mathcal{M}\left(u ; \sigma_{0}^{p}, \sigma_{0}^{b}\right)|\dot{\xi}|,
$$

where $\theta=\sigma_{d}$ respectively $\theta=2 \mu \varepsilon_{d}$,

$$
\mathcal{M}\left(u ; \sigma_{0}^{p}, \sigma_{0}^{b}\right)(t)=\frac{1}{C} \int_{I} \gamma(k) \sigma_{k}^{b}(t) d \nu(k),
$$

and $(2.43)-(2.46)$ are used to express $\sigma_{k}^{b}$ in terms of the arguments of $\mathcal{M}$. Equation (2.47) is complemented by the initial condition

$$
u\left(t_{0}\right)=C \varepsilon^{p}\left(t_{0}\right)+\sigma_{0 d}^{p} .
$$

In the stress controlled case, $\varepsilon^{p}\left(t_{0}\right)$ is prescribed, whereas in the strain controlled case, it can be expressed in terms of the given data by (2.38).

Once the auxiliary equation (2.47) is solved, we can express the operators $\mathcal{F}, \mathcal{G}$ in terms of $u$, namely

$$
\begin{gathered}
\varepsilon=\mathcal{F}\left(\sigma, \sigma_{0}^{p}, \sigma_{0}^{b}, \varepsilon_{0}^{p}\right)=\varepsilon^{e}+\varepsilon^{p}=A^{-1} \sigma+\frac{1}{C} \mathcal{P}\left(u ; \sigma_{0 d}^{p}\right), \\
\sigma=\mathcal{G}\left(\varepsilon, \sigma_{0}^{p}, \sigma_{0}^{b}\right)=A\left(\varepsilon-\varepsilon^{p}\right)=A \varepsilon-\frac{2 \mu}{C} \mathcal{P}\left(u ; \sigma_{0 d}^{p}\right)
\end{gathered}
$$




\section{Proof of the Wellposedness}

The wellposedness of the initial value problem

$$
\begin{gathered}
\dot{u}(t)=\dot{\theta}(t)+\mathcal{M}\left(u ; \sigma_{0}^{p}, \sigma_{0}^{b}\right)(t)|\dot{\xi}(t)|, \quad \xi(t)=\mathcal{P}\left(u ; \sigma_{0 d}^{p}\right)(t), \\
u\left(t_{0}\right)=u^{0} .
\end{gathered}
$$

has been studied in [2] concerning the dependence on $\theta$; the dependence on the initial conditions $\left(u^{0}, \sigma_{0}^{p}, \sigma_{0}^{b}\right)$ does not pose any new problems. For the convenience of the reader, we repeat the formulation of the existence theorem, adapted to the present case.

Theorem 3.1 Let $\theta \in W^{1,1}\left(t_{0}, t_{1} ; \mathbb{T}_{d}\right), u^{0} \in \mathbb{T}_{d}$ and an operator

$$
\mathcal{M}: C\left(\left[t_{0}, t_{1}\right] ; \mathbb{T}_{d}\right) \times \mathbb{T}_{r}^{p} \times \mathcal{T}^{b} \rightarrow C\left(\left[t_{0}, t_{1}\right] ; \mathbb{T}_{d}\right)
$$

be given. Assume that $\mathcal{M}\left(\cdot ; \sigma_{0}^{p}, \sigma_{0}^{b}\right)$ is causal and continuous with respect to the maximum norm for all $\sigma_{0}^{p} \in \mathbb{T}_{r}^{p}$ and $\sigma_{0}^{b} \in \mathcal{T}^{b}$, and that $\kappa>0, \sigma_{0}^{p} \in \mathbb{T}_{r}^{p}, \sigma_{0}^{b} \in \mathcal{T}^{b}$ and $u^{0} \in \mathbb{T}_{d}$ are given such that

$$
\sup _{\tau \in\left[t_{0}, t\right]}\left|\mathcal{M}\left(u ; \sigma_{0}^{p}, \sigma_{0}^{b}\right)\right| \leq 1-\kappa
$$

holds for all $t \in\left[t_{0}, t_{1}\right]$ and all $u \in W^{1,1}\left(t_{0}, t ; \mathbb{T}_{d}\right)$ with $u\left(t_{0}\right)=u^{0}$ and

$$
|\dot{u}(\tau)| \leq \frac{1}{\kappa}|\dot{\theta}(\tau)|, \quad \text { a.e. in }\left(t_{0}, t\right) .
$$

Then there exists a solution $(u, \xi)$ of the Cauchy problem (3.1), (3.2) where the functions $u, \xi \in W^{1,1}\left(t_{0}, t_{1} ; \mathbb{T}_{d}\right)$ fulfil (3.4) and (3.5). Moreover, every such solution which satisfies (3.4) also satisfies (3.5).

Proof. See [2], Theorem 3.2.

Lemma 3.2 The operator $\mathcal{M}\left(\cdot ; \sigma_{0}^{p}, \sigma_{0}^{b}\right)$ as defined in (2.43) - (2.48) is causal and continuous on $C\left(\left[t_{0}, t_{1}\right] ; \mathbb{T}_{d}\right)$ for all $\sigma_{0}^{p} \in \mathbb{T}_{r}^{p}$ and $\sigma_{0}^{b} \in \mathcal{T}^{b}$. The backstresses $\sigma_{k}^{b}$ satisfy the a priori estimate

$$
\left|\sigma_{k}^{b}(t)\right| \leq R(k), \quad \text { a.e. in }\left(t_{0}, t_{1}\right),
$$

for all $k \in I$.

Proof. The estimate (3.6) follows from the variations of constants formula (2.46), since $|x(t)| \leq r$ and $\left|\sigma_{0}^{b}(k)\right| \leq R(k)$ hold for all $t$ and $k$. Let now $u_{n} \in C\left(\left[t_{0}, t_{1}\right] ; \mathbb{T}_{d}\right)$ converge uniformly to $u \in C\left(\left[t_{0}, t_{1}\right] ; \mathbb{T}_{d}\right)$. It is known (see [9]) that

$$
\begin{gathered}
\xi_{n}=\mathcal{P}\left(u_{n} ; \sigma_{0 d}^{p}\right) \rightarrow \xi=\mathcal{P}\left(u ; \sigma_{0 d}^{p}\right), \quad x_{n}=\mathcal{S}\left(u_{n} ; \sigma_{0 d}^{p}\right) \rightarrow x=\mathcal{S}\left(u ; \sigma_{0 d}^{p}\right), \\
V_{n}(t)=\operatorname{Var}_{\left[t_{0}, t\right]} \xi_{n} \rightarrow V(t)=\operatorname{Var}_{\left[t_{0}, t\right]} \xi,
\end{gathered}
$$

uniformly on $\left[t_{0}, t_{1}\right]$. An application of Lebesgue's dominated convergence theorem yields the assertion.

We now discuss the boundedness property (3.4). By the definition of $\mathcal{M}$ in (2.48), the estimate (3.6) yields

$$
\left\|\mathcal{M}\left(u ; \sigma_{0}^{p}, \sigma_{0}^{b}\right)\right\|_{\infty} \leq \frac{\Gamma_{1}}{C}
$$


so (3.4) holds for all arguments, regardless of (3.5), with

$$
\kappa=\frac{\nu^{l} C^{l}}{C}, \quad \text { respectively } \quad \kappa=\frac{2 \mu+\nu^{l} C^{l}}{C},
$$

in the stress respectively strain controlled case. Thus, the existence of a solution of (3.1), (3.2) follows for the strain controlled case and, if in addition $\nu^{l}>0$, also for the stress controlled case.

Existence proof for the stress controlled case with $\nu^{l}=0$. Let $\kappa>0$. According to Theorem 2.4, we want to prove existence for initial conditions satisfying

$$
\begin{gathered}
\sigma\left(t_{0}\right)=\sigma_{0}^{p}+\int_{I} \sigma_{0}^{b}(k) d \nu(k), \\
\left|\int_{I} \gamma(k) \sigma_{0}^{b}(k) d \nu(k)\right| \leq \Gamma_{1}(1-\kappa),
\end{gathered}
$$

and for stress inputs $\sigma_{d} \hat{=} \theta \in W^{1,1}\left(t_{0}, t_{1} ; \mathbb{T}_{d}\right)$ satisfying

$$
\left\|\sigma_{d}\right\|_{\infty} \leq \Gamma_{0}+r-\Gamma_{1} \beta \kappa, \quad \beta=\frac{1}{\gamma_{\min }}+\frac{r \gamma_{\max }}{\Gamma_{2}} .
$$

Let such a $\sigma_{d}$ be given, choose $\eta>0$ small enough such that

$$
\int_{t}^{t+\eta}\left|\dot{\sigma}_{d}(\tau)\right| d \tau \leq \frac{\kappa^{2} \Gamma_{1}}{8 \gamma_{\max }}, \quad \forall t \in\left[t_{0}, t_{1}-\eta\right]
$$

In the first step we will prove that, if we have a solution $u$ of (3.1), (3.2) satisfying (3.4) on $\left[t_{0}, a\right]$, then it can be extended to $[a, a+\eta]$, and every such extension $\tilde{u}$ satisfies

$$
\left\|\mathcal{M}\left(\tilde{u} ; \sigma_{0}^{p}, \sigma_{0}^{b}\right)\right\|_{\infty} \leq 1-\frac{\kappa}{2}
$$

on $[a, a+\eta]$, and

$$
|\dot{\tilde{u}}(t)| \leq \frac{2}{\kappa}\left|\dot{\sigma}_{d}(t)\right|, \quad \text { a.e. on }(a, a+\eta)
$$

To this end, let $\tilde{u} \in W^{1,1}\left(a, a+\eta ; \mathbb{T}_{d}\right)$ be an arbitrary function which satisfies (3.16) as well as $\tilde{u}(a)=u(a)$; setting $\tilde{u}=u$ on $\left[t_{0}, a\right]$ we may regard it as an element of $W^{1,1}\left(t_{0}, a+\eta ; \mathbb{T}_{d}\right)$ as well. From the variation of constants formula (2.46), applied on the interval $[a, a+\eta]$, we obtain

$$
\left|\sigma_{k}^{b}(t)-\sigma_{k}^{b}(a)\right| \leq\left(1-\exp \left(-\frac{\gamma(k)}{\Gamma_{1}}(V(t)-V(a))\right)\right) \cdot\left(\left|\sigma_{k}^{b}(a)\right|+R(k)\right), \quad t \in[a, a+\eta],
$$

for the corresponding backstresses. Since

$$
|V(t)-V(a)| \leq \int_{a}^{t}|\dot{\tilde{u}}(\tau)| d \tau,
$$

we get

$$
\begin{aligned}
\left|\sigma_{k}^{b}(t)-\sigma_{k}^{b}(a)\right| & \leq 2 R(k) \frac{\gamma_{\max }}{\Gamma_{1}} \int_{a}^{t}|\dot{\tilde{u}}(\tau)| d \tau \leq \frac{4 \gamma_{\max }}{\kappa C_{1}} R(k) \int_{a}^{a+\eta}\left|\dot{\sigma}_{d}(\tau)\right| d \tau \\
& \leq \frac{\kappa}{2} R(k)
\end{aligned}
$$


SO

$$
\left|\mathcal{M}\left(\tilde{u} ; \sigma_{0}^{p}, \sigma_{0}^{b}\right)(t)-\mathcal{M}\left(\tilde{u} ; \sigma_{0}^{p}, \sigma_{0}^{b}\right)(a)\right| \leq \frac{\kappa}{2} .
$$

Thus, the assumption $\left|\mathcal{M}\left(\tilde{u} ; \sigma_{0}^{p}, \sigma_{0}^{b}\right)(a)\right| \leq 1-\kappa$ implies that (3.15) holds if $\tilde{u}$ satisfies (3.16). We may therefore apply Theorem 3.1 on the interval $[a, a+\eta]$ to conclude the first step of the proof. In the second step, we use (3.13) to show that (3.15) can be improved to

$$
\left|\mathcal{M}\left(\tilde{u} ; \sigma_{0}^{p}, \sigma_{0}^{b}\right)(t)\right| \leq 1-\kappa, \quad \forall t \in[a, a+\eta] .
$$

In fact, if (3.21) does not hold, then there must exist a $t \in(a, a+\eta)$ such that

$$
\left|\mathcal{M}\left(\tilde{u} ; \sigma_{0}^{p}, \sigma_{0}^{b}\right)(t)\right|>1-\kappa, \quad \frac{\mathrm{d}}{\mathrm{dt}}\left(\left|\mathcal{M}\left(\tilde{u} ; \sigma_{0}^{p}, \sigma_{0}^{b}\right)(t)\right|^{2}\right)>0
$$

Let us define

$$
\alpha=\left|\mathcal{M}\left(\tilde{u} ; \sigma_{0}^{p}, \sigma_{0}^{b}\right)(t)\right|, \quad e=\frac{1}{\alpha} \mathcal{M}\left(\tilde{u} ; \sigma_{0}^{p}, \sigma_{0}^{b}\right)(t) \in \mathbb{T}_{d}
$$

then obviously

$$
0<1-\alpha<\kappa, \quad|e|=1
$$

The choice of $t$ implies that

$$
\begin{aligned}
0 & <\frac{1}{2} \frac{\mathrm{d}}{\mathrm{dt}}\left(\left|\mathcal{M}\left(\tilde{u} ; \sigma_{0}^{p}, \sigma_{0}^{b}\right)(t)\right|^{2}\right)=\frac{\alpha}{\Gamma_{1}} \int_{I} \gamma(k)\left\langle\dot{\sigma}_{k}^{b}(t), e\right\rangle d \nu(k) \\
& =\frac{\alpha}{\Gamma_{1}}|\dot{\xi}(t)| \int_{I} \gamma(k)^{2}\left\langle\frac{R(k)}{r} x(t)-\sigma_{k}^{b}(t), e\right\rangle d \nu(k)
\end{aligned}
$$

so in particular $|\dot{\xi}(t)|>0$ and therefore

$$
\int_{I} \gamma(k)^{2}\left\langle\sigma_{k}^{b}(t), e\right\rangle d \nu(k)<\frac{\Gamma_{2}}{r}\langle x(t), e\rangle=\frac{\Gamma_{2}}{r}\left(\left\langle\sigma_{d}(t), e\right\rangle-\int_{I}\left\langle\sigma_{k}^{b}(t), e\right\rangle d \nu(k)\right)
$$

hence

$$
\int_{I}\left(\gamma(k)^{2}+\frac{\Gamma_{2}}{r}\right)\left\langle\sigma_{k}^{b}(t), e\right\rangle d \nu(k)<\frac{\Gamma_{2}}{r}\left\|\sigma_{d}\right\|_{\infty} .
$$

On the other hand, the a priori estimate $\left|\sigma_{k}^{b}(t)\right| \leq R(k)$ shows that

$$
0<1-\alpha=\frac{1}{\Gamma_{1}} \int_{I} \gamma(k)\left\langle R(k) e-\sigma_{k}^{b}(t), e\right\rangle d \nu(k)<\kappa,
$$

hence the definition of $\beta$ in (3.13) yields

$$
\begin{aligned}
\int_{I}\left(1+\frac{r}{\Gamma_{2}} \gamma(k)^{2}\right)\left\langle R(k) e-\sigma_{k}^{b}(t), e\right\rangle d \nu(k) & \leq \beta \int_{I} \gamma(k)\left\langle R(k) e-\sigma_{k}^{b}(t), e\right\rangle d \nu(k) \\
& \leq \beta \Gamma_{1} \kappa
\end{aligned}
$$

and therefore

$$
\begin{aligned}
\left\|\sigma_{d}\right\|_{\infty}> & \int_{I}\left(1+\frac{r}{\Gamma_{2}} \gamma(k)^{2}\right) R(k) d \nu(k) \\
& \quad-\int_{I}\left(1+\frac{r}{\Gamma_{2}} \gamma(k)^{2}\right)\left\langle R(k) e-\sigma_{k}^{b}(t), e\right\rangle d \nu(k) \\
\geq & \Gamma_{0}+r-\beta \Gamma_{1} \kappa
\end{aligned}
$$


which contradicts our assumption (3.13). Thus, such a $t$ cannot exist, and the second step is proved. Applying the two steps in an alternate fashion we are able to cover the whole interval $\left[t_{0}, t_{1}\right]$, thus completing the existence proof.

Proof of uniqueness and Lipschitz continuous dependence. We combine a Gronwall type argument with the Lipschitz continuity property of the hysteresis operators $\mathcal{P}$ and $\mathcal{S}$. As the arguments are essentially the same as for the single surface case, i.e. the model of Armstrong and Frederick, we can use the results of [2] to a large extent.

Proposition 3.3 Let two sets of data $\left(\theta_{1}, u_{1}^{0}, \sigma_{10}^{p}, \sigma_{10}^{b}\right),\left(\theta_{2}, u_{2}^{0}, \sigma_{20}^{p}, \sigma_{20}^{b}\right)$ with $\theta_{i} \in \Theta$, $u_{i}^{0} \in X, \sigma_{i 0}^{p} \in \mathbb{T}_{r}^{p}$ and $\sigma_{i 0}^{b} \in \mathcal{T}^{b}$ be given, let $\left(u_{1}, \xi_{1}\right)$ and $\left(u_{2}, \xi_{2}\right)$ be corresponding solutions in $W^{1,1}\left(t_{0}, t_{1} ; \mathbb{T}_{d}\right)$ of the Cauchy problem (3.1), (3.2) which satisfy (3.4) and (3.5). Assume that

$$
\begin{aligned}
& \max _{s \in\left[t_{0}, t\right]}\left|\mathcal{M}\left(u_{1} ; \sigma_{10}^{p}, \sigma_{10}^{b}\right)(s)-\mathcal{M}\left(u_{2} ; \sigma_{20}^{p}, \sigma_{20}^{b}\right)(s)\right| \leq A\left(\left|\sigma_{10}^{p}-\sigma_{20}^{p}\right|+\left\|\sigma_{10}^{b}-\sigma_{20}^{b}\right\|_{L_{\nu}^{1}\left(I ; \mathbb{T}_{d}\right)}\right. \\
& \left.\quad+\left|u_{1}^{0}-u_{2}^{0}\right|+\int_{t_{0}}^{t}\left|\dot{u}_{1}-\dot{u}_{2}\right| d s\right)
\end{aligned}
$$

holds for all $t \in\left[t_{0}, t_{1}\right]$. Then there holds

$$
\left\|u_{1}-u_{2}\right\|_{1,1} \leq L\left(\left|u_{1}^{0}-u_{2}^{0}\right|+\left|\sigma_{10}^{p}-\sigma_{20}^{p}\right|+\left\|\sigma_{10}^{b}-\sigma_{20}^{b}\right\|_{L_{\nu}^{1}\left(I ; \mathbb{T}_{d}\right)}+\left\|\theta_{1}-\theta_{2}\right\|_{1,1}\right),
$$

where $L$ depends only upon $A, \kappa, r$ and

$$
c:=\max \left\{\left\|\theta_{1}\right\|_{1,1},\left\|\theta_{2}\right\|_{1,1}\right\} .
$$

Proof. See Theorem 3.3 in [2].

The operator $\mathcal{M}$ as defined by (2.43) - (2.48) satisfies

$$
\begin{aligned}
& \left|\mathcal{M}\left(u_{1} ; \sigma_{10}^{p}, \sigma_{10}^{b}\right)(t)-\mathcal{M}\left(u_{2} ; \sigma_{20}^{p}, \sigma_{20}^{b}\right)(t)\right| \leq \int_{I} \gamma(k)\left|\sigma_{10}^{b}(k)-\sigma_{20}^{b}(k)\right| d \nu(k) \\
& \quad+\left(\frac{2 \Gamma_{2}}{C}+\frac{\Gamma_{3}}{C^{2}} \int_{t_{0}}^{t}\left|\dot{\xi}_{1}(s)\right| d s\right) \int_{t_{0}}^{t}\left|\dot{\xi}_{1}-\dot{\xi}_{2}\right| d s
\end{aligned}
$$

as a repeated use of the triangle inequality as well as of the inequality $\mid \exp (-t)-$ $\exp (-s)|\leq| t-s \mid$, valid for $t, s \geq 0$, shows. It was proved in [2], Theorem A.5, that

$$
\int_{t_{0}}^{t}\left|\dot{\xi}_{1}-\dot{\xi}_{2}\right| d s \leq\left|\sigma_{10 d}^{p}-\sigma_{20 d}^{p}\right|+\int_{t_{0}}^{t}\left|\dot{u}_{1}-\dot{u}_{2}\right| d s+\frac{\sqrt{2}}{r} \int_{t_{0}}^{t}\left|\dot{u}_{1}\right|\left|x_{1}-x_{2}\right| d s
$$

holds. Moreover, by the standard uniqueness argument for variational inequalities (see also Proposition A.1 in [2]), one has

$$
\left|x_{1}(t)-x_{2}(t)\right| \leq\left|\sigma_{10 d}^{p}-\sigma_{20 d}^{p}\right|+\int_{t_{0}}^{t}\left|\dot{u}_{1}-\dot{u}_{2}\right| d s .
$$

Putting together the estimates (3.34) - (3.36), one sees that $\mathcal{M}$ satisfies the assumption (3.31) with some constant $A$ which depends only on $\left\|u_{1}\right\|_{1,1},\left\|u_{2}\right\|_{1,1}$ and on the problem data. Therefore the Lipschitz estimate (3.32) holds for the difference $u_{1}-u_{2}$ of the two solutions. It extends to all the unknown functions in the Chaboche model, since they can be expressed in terms of $u$ and $\xi$ as shown at the end of Section 2, both for the stress controlled and the strain controlled case. Thus, the proof of Theorems 2.3 and 2.4 is complete. 


\section{References}

[1] P.J. ARMSTRONG and C.O. FREDERICK, 1966, A mathematical representation of the multiaxial Bauschinger effect, C.E.G.B., Report RD/B/N 731.

[2] M. BROKATE, P. KREJČÍ, Wellposedness of kinematic hardening models in elastoplasticity, Math. Model. Numer. Anal., to appear.

[3] M. BROKATE, J. SPREKELS, 1996, Hysteresis and phase transitions, SpringerVerlag, Berlin.

[4] J.-L. CHABOCHE, 1989, Constitutive equations for cyclic plasticity and cyclic viscoplasticity, Int. J. Plasticity, 5, pp. $247-302$.

[5] J.-L. CHABOCHE, 1991, On some modifications of kinematic hardening to improve the description of ratchetting effects, Int. J. Plasticity, 7, pp. $661-678$.

[6] J.-L. CHABOCHE, 1994, Modeling of ratchetting: evaluation of various approaches, Eur. J. Mech., A/Solids, 13 , pp. $501-518$.

[7] M. KAMLAH, M. KORZEŃ and CH. TSAKMAKIS, Uniaxial ratchetting in rateindependent plasticity laws, Acta Mechanica, to appear.

[8] M.A. KRASNOSEL'SKII and A.V. POKROVSKII, 1989, Systems with hysteresis, Springer-Verlag, Berlin. Russian edition: Nauka, Moscow 1983.

[9] P. KREJČÍ, 1996, Hysteresis, convexity and dissipation in hyperbolic equations, Gakkotosho, Tokyo.

[10] J. LEMAITRE and J.-L. CHABOCHE, 1990, Mechanics of solid materials, Cambridge University Press, Cambridge 1990. French edition: Dunod, Paris 1985.

[11] G.A. MAUGIN, 1992, The thermomechanics of plasticity and fracture, Cambridge University Press, Cambridge 1992.

[12] E. MELAN, 1938, Zur Plastizität des räumlichen Kontinuums, Ingenieur-Archiv, 9, $116-126$.

[13] W. PRAGER, 1949, Recent developments in the mathematical theory of plasticity, J. Appl. Phys., 20 , pp. $235-241$.

[14] A. VISINTIN, 1994, Differential models of hysteresis, Springer-Verlag, Berlin. 